\title{
Boundary Condition in the Oscillating Turbulent Boundary Layer for the Simulation of Wave Breaking
}

\author{
Benedetta lele \\ Department of Civil, Constructional and Environmental \\ Engineering "Sapienza" University of Rome \\ Via Eudossiana 18, 00184 \\ ITALY \\ +390644585062 \\ benedetta.iele@uniroma1.it
}

\author{
Federica Palleschi \\ Department of Civil, Constructional and Environmental \\ Engineering "Sapienza" University of Rome \\ Via Eudossiana 18, 00184 \\ ITALY \\ +390644585062 \\ federica.palleschi@uniroma1.it
}

\begin{abstract}
In this paper a new numerical model for the simulation of the wave breaking is proposed. In order to represent the complex geometry of coastal regions, the three-dimensional equations of motion are expressed in integral contravariant form and are solved on a curvilinear boundary conforming grid. A time-dependent transformation of the vertical coordinate that is a function of the oscillation of the turbulent wave boundary layer is proposed. New boundary condition bottom for the equations of motion expressed in contravariant form are proposed. In order to correctly simulate the height of the breaking waves, the importance of the correct positioning, inside the oscillating turbulent boundary layer, of the centre of the calculation grid cell closest to the bottom, is demonstrated.
\end{abstract}

\section{CCS Concepts}

-Applied Computing Physical Sciences and Engineering $\rightarrow$ Engineering.

\section{Keywords}

Wave breaking; three-dimensional model; integral contravariant form; time-dependent curvilinear coordinate system; boundary conditions; moving grids in boundary layer.

\section{INTRODUCTION}

In hydraulic engineering, the simulation of the hydrodynamic fields and turbulence under breaking waves allow the analysis of the effect produced by coastal defense structures and the modifications of the shoreline. The two-dimensional depthaveraged equations of motion [1-5] is one of the most used approaches for the simulation of breaking waves. This approach assumes a simplified distribution of the hydrodynamic quantities along the vertical direction (depth averaged models). In the literature, for the three-dimensional simulation of wave induced free surface flows many authors adopt numerical models that integrate the three-dimensional Navier- Stokes equations, in which the so-called $\sigma$ transformation is used. In such a

Permission to make digital or hard copies of part or all of this work for personal or classroom use is granted without fee provided that copies are not made or distributed for profit or commercial advantage and that copies bear this notice and the full citation on the first page. Copyrights for third-party components of this work must be honored. For all other uses, contact the Owner/Author.

ICCTA '20, April 14-16, 2020, Antalya, Turkey

(C) 2020 Copyright is held by the owner/author(s).

ACM ISBN 978-1-4503-7749-2/20/04 ...\$15.00

https://doi.org/10.1145/3397125.3397131 framework, the vertical Cartesian coordinate is transformed in a vertical coordinate that moves with the free surface [6-7]. Other authors [8] recently express the three-dimensional equations of motion in a boundary conforming curvilinear coordinate system, where the vector and tensor quantities are expressed in a Cartesian frame of reference and only the vertical coordinate varies over time.

In this paper, we propose a time-dependent transformation of the vertical coordinate that is a function also of the height of the oscillating turbulent wave boundary layer. In the literature, there is a contradiction in the way to assign the boundary conditions at the bottom. As it is known, the turbulent boundary layer can be subdivided in three regions: the viscous sub-layer is the region closest to the bottom and is characterized by the dominance of the viscous stress; the buffer layer is the intermediate region and is characterized by the equal importance of viscous and turbulent stresses; the turbulent core is the region which is further from the bottom and where the turbulent stresses are dominant.

In the literature, the velocity boundary conditions, the friction velocity in the turbulent wave boundary layer are deduced from appropriate logarithmic law.

The numerical solution of the momentum equation allows us to determine the cell-averaged velocity in the centre of the calculation cell closest to a bottom. Using the cell-averaged velocity, it is possible to calculate the friction velocity and the velocity boundary condition by the logarithmic law. The centre of the calculation cell closest to the bottom (where the cell-averaged velocity is calculated by the momentum equation) must necessarily be located inside of the turbulent core, because the logarithmic law is valid just in the turbulent core.

Wrong evaluations of the friction velocity, velocity boundary conditions and turbulent stresses in the turbulent boundary layer are produced by placing the centre of the calculation cell closest to the bottom outside the boundary layer, or too close to the bottom (in the buffer layer or even in the viscous sub-layer).

The above-mentioned centre of the calculation cell closest to the bottom oscillates coherently with the oscillations of the freesurface, according to the current numerical models that use $\sigma$ coordinate transformation. This oscillation is in contradictory form with respect to the oscillations of the turbulent wave boundary layer. Consequently, during the wave period, the centre of the calculation cell closest to the bottom is located outside or inside the turbulent wave boundary layer. By using these numerical models, wrong evaluations of velocity boundary conditions, friction velocity and bottom stresses occur, 
consequently wrong simulation of the wave height at the breaking point and in the surf-zone occur.

In this work, a new model for the simulation of breaking waves is proposed. The three-dimensional equations of motion are expressed in integral contravariant form in time varying coordinates that can adapt to free-surface movements and to the evolution of the turbulent boundary layer thickness and are solved on boundary conforming curvilinear grids that can reproduce the complex geometry of the coastal regions.

New boundary condition at the bottom for the contravariant equations is proposed. In order to correctly simulate the height of the breaking wave, we present an analysis about the importance of the correct positioning, inside the oscillating turbulent boundary layer, of the centre of the calculation cell closest to the bottom.

In this work, the centre of the calculation cell closest to the bottom oscillates coherently with the oscillations of the turbulent wave boundary layer and is always located in the turbulent core. On the lower face of the calculation cell closest to the bottom, the boundary condition for the cell averaged flow velocity, that is deduced form the logarithmic law, and for the eddy viscosity (which intervenes in the closure relation for the turbulent stress tensor in the turbulent boundary layer) are assigned. The abovementioned lower face of the calculation cell closest to the bottom is in the lower part of the turbulent core, close to the buffer layer, where the balance between the production and dissipation of turbulent kinetic energy take place.

\section{GOVERNING EQUATIONS}

In this paper we adopt the governing equations proposed in [9-10] in which the Navier-Stokes equations are expressed in integral contravariant form in a time-dependent curvilinear coordinate system. Let be $u^{k}(k=1,3)$ is the contravariant component of the fluid velocity; $v^{\alpha}(\alpha=1,3)$ is the contravariant component of the velocity of the moving coordinate lines; $\rho$ is the water density; $f^{k}$ and $R^{k \alpha} \quad(k, \alpha=1,3)$ are, respectively, the contravariant component of the external body forces for unit mass vector and the contravariant turbulent stress tensor.

$$
\begin{gathered}
\frac{d}{d \tau} \int_{\Delta V_{0}}\left(\overrightarrow{\tilde{g}}^{(l)} \cdot \vec{g}_{(k)} \rho u^{k} \sqrt{g}\right) d \xi^{1} d \xi^{2} d \xi^{3}+ \\
\sum_{\alpha=1}^{3}\left\{\int_{\Delta A_{0}^{\alpha+}}\left(\overrightarrow{\tilde{g}}^{(l)} \cdot \vec{g}_{(k)} \rho u^{k}\left(u^{\alpha}-v^{\alpha}\right) \sqrt{g}\right) d \xi^{\beta} d \xi^{\gamma}-\right. \\
\left.\int_{\Delta A_{0}^{\alpha-}}\left(\overrightarrow{\tilde{g}}^{(l)} \cdot \vec{g}_{(k)} \rho u^{k}\left(u^{\alpha}-v^{\alpha}\right) \sqrt{g}\right) d \xi^{\beta} d \xi^{\gamma}\right\}= \\
\int_{\Delta V_{0}}\left(\overrightarrow{\tilde{g}}^{(l)} \cdot \vec{g}_{(k)} \rho f^{k} \sqrt{g}\right) d \xi^{1} d \xi^{2} d \xi^{3}+ \\
\sum_{\alpha=1}^{3}\left\{\int_{\Delta A_{0}^{\alpha+}}\left(\overrightarrow{\tilde{g}}^{(l)} \cdot \vec{g}_{(k)} R^{k \alpha} \sqrt{g}\right) d \xi^{\beta} d \xi^{\gamma}-\right. \\
\left.\int_{\Delta A_{0}^{\alpha-}}\left(\overrightarrow{\tilde{g}}^{(l)} \cdot \vec{g}_{(k)} R^{k \alpha} \sqrt{g}\right) d \xi^{\beta} d \xi^{\gamma}\right\}
\end{gathered}
$$

$$
\begin{gathered}
\frac{d}{d \tau} \int_{\Delta V_{0}}(\rho \sqrt{g}) d \xi^{1} d \xi^{2} d \xi^{3}+ \\
\sum_{\alpha=1}^{3}\left\{\int_{\Delta A_{0}^{\alpha+}}\left(\rho\left(u^{\alpha}-v^{\alpha}\right) \sqrt{g}\right) d \xi^{\beta} d \xi^{\gamma}-\right. \\
\left.\int_{\Delta A_{0}^{\alpha-}}\left(\rho\left(u^{\alpha}-v^{\alpha}\right) \sqrt{g}\right) d \xi^{\beta} d \xi^{\gamma}\right\}=0
\end{gathered}
$$

In the above equations $\tau$ is the time and $\xi^{1}, \xi^{2}, \xi^{3}$ are moving curvilinear coordinates obtained from the Cartesian coordinate system $\left(x^{1}, x^{2}, x^{3}, t\right)$ by a time-dependent transformation $x^{i}=$ $x^{i}\left(\xi^{1}, \xi^{2}, \xi^{3}, \tau\right), t=\tau$. Let $\vec{g}_{(l)}$ and $\vec{g}^{(l)}$ be, respectively, the covariant and contravariant base vectors of the curvilinear coordinate system; $\sqrt{g}$ the Jacobian of the transformation. $\Delta V_{0}=$ $\Delta \xi^{1} \Delta \xi^{2} \Delta \xi^{3}$ is the volume element in the transformed space and $\Delta A_{0}^{\alpha+}$ and $\Delta A_{0}^{\alpha-}$ indicate the contour surfaces of the volume $\Delta V_{0}$ on which $\xi^{\alpha}$ is constant and which are located at the larger and at the smaller value of $\xi^{\alpha}$ respectively. Here, the indexes $\alpha, \beta$, and $\gamma$ are cyclic.

The general integral form of the Navier-Stokes equations expressed in a time dependent curvilinear coordinate system are represented by equation (1) and (2). The complete derivation of these equations can be found in [10]. The integral Equations (1) and (2) are reduced to the complete differential form of the contravariant Navier-Stokes equations in a time dependent curvilinear coordinate system that have been proposed in the literature by Luo and Bewley [11] by taking the limit as the volume approaches zero, as it has been demonstrate in [9].

In this paper, we start from the model proposed in [9-10] and obtain the following governing equations in order to simulate the fully dispersive wave processes and the wave breaking

$$
\begin{gathered}
\frac{\partial \overline{H u l}}{\partial \tau}=- \\
\frac{1}{\Delta V_{0} \sqrt{g_{0}}} \sum_{\alpha=1}^{3}\left\{\int _ { \Delta A _ { o } ^ { \alpha + } } \left[\overrightarrow{\tilde{g}}^{(l)} \cdot \vec{g}_{(k)} H u^{k}\left(u^{\alpha}-v^{\alpha}\right)+\overrightarrow{\tilde{g}}^{(l)}\right.\right. \\
\left.\cdot \vec{g}^{(\alpha)} G H^{2}\right] \sqrt{g_{0}} d \xi^{\beta} d \xi^{\gamma}- \\
\int_{\Delta A_{o}^{\alpha-}}\left[\overrightarrow{\tilde{g}}^{(l)} \cdot \vec{g}_{(k)} H u^{k}\left(u^{\alpha}-v^{\alpha}\right)+\overrightarrow{\tilde{g}}^{(l)}\right. \\
\frac{1}{\Delta V_{0} \sqrt{g_{0}}} \sum_{\alpha=1}^{3}\left\{\int_{\Delta A_{o}^{\alpha+}} \overrightarrow{\tilde{g}}^{(l)} \cdot \vec{g}^{(\alpha)} G h H \sqrt{g_{0}} d \xi^{\beta} d \xi^{\gamma}-\right. \\
\left.\int_{\Delta A_{o}^{\alpha-}} \overrightarrow{\tilde{g}}^{(l)} \cdot \vec{g}^{(\alpha)} G h H \sqrt{g_{0}} d \xi^{\beta} d \xi^{\gamma}\right\}+ \\
\frac{1}{\Delta V_{0} \sqrt{g_{0}}} \sum_{\alpha=1}^{3}\left\{\int_{\Delta A_{o}^{\alpha+}} \overrightarrow{\tilde{g}}^{(l)} \cdot \vec{g}_{(k)} \frac{T^{k \alpha}}{\rho} H \sqrt{g_{0}} d \xi^{\beta} d \xi^{\gamma}-\right. \\
\left.\int_{\Delta A_{o}^{\alpha-}} \overrightarrow{\tilde{g}}^{(l)} \cdot \vec{g}_{(k)} \frac{R^{k \alpha}}{\rho} H \sqrt{g_{0}} d \xi^{\beta} d \xi^{\gamma}\right\}- \\
\frac{1}{\Delta V_{0} \sqrt{g_{0}}} \int_{\Delta V_{0}} \overrightarrow{\tilde{g}}^{(l)} \cdot \vec{g}^{(m)} \frac{\partial p_{d}}{\partial \xi^{m}} H \sqrt{g_{0}} d \xi^{1} d \xi^{2} d \xi^{3}
\end{gathered}
$$




$$
\begin{array}{r}
\frac{\partial \bar{H}}{\partial \tau}=\frac{1}{\Delta A_{o}^{3} \sqrt{g_{0}}} \sum_{\alpha=1}^{2}\left[\int_{0}^{1} \int_{\Delta \xi_{o}^{\alpha+}} u^{\alpha} H \sqrt{g_{0}} d \xi^{\beta} d \xi^{3}\right. \\
\left.-\int_{0}^{1} \int_{\Delta \xi_{o}^{\alpha-}} u^{\alpha} H \sqrt{g_{0}} d \xi^{\beta} d \xi^{3}\right]
\end{array}
$$

where $H=h+\eta$ is the total water depth $H=h+\eta ; h$ is the undisturbed water depth and $\eta$ is the free surface elevation with respect to the undisturbed water level; $G$ is the gravity acceleration; pressure $p$ is divided into a hydrostatic part, $\rho G\left(\eta-x^{3}\right)$ (the vertical coordinate $x^{3}$ is zero at the still free surface and it is positive upwards), and a dynamic one, $p_{d}$. The curvilinear coordinates $\xi^{1}, \xi^{2}, \xi^{3}, \tau$ are defined as

$$
\begin{gathered}
\xi^{1}=\xi^{1}\left(x^{1}, x^{2}, x^{3}\right) \quad ; \quad \xi^{2}=\xi^{2}\left(x^{1}, x^{2}, x^{3}\right) \quad ; \\
\xi^{3}=\frac{x^{3}+h\left(x^{1}, x^{2}\right)}{H\left(x^{1}, x^{2}, x^{3}, t\right)} \quad ; \quad \tau=t
\end{gathered}
$$

where $\xi^{1}$ and $\xi^{2}$ are the horizontal boundary conforming curvilinear coordinates and $\xi^{3}$ is the time varying vertical coordinate by which the irregular varying domain in the physical space is mapped into a regular fixed domain in the transformed space. $\sqrt{g}=\vec{k} \cdot\left|\vec{g}_{(1)} \wedge \vec{g}_{(2)}\right|$, where $\wedge$ indicates the vector product. $\bar{H}$ and $\overline{H u^{l}}$ are spatial average values over volume elements defined in the form

$$
\begin{gathered}
\bar{H}=\frac{1}{\Delta A_{0}^{3} \sqrt{g_{0}}} \int_{\Delta A_{o}^{3}} H \sqrt{g_{0}} d \xi^{1} d \xi^{2} \\
\overline{H u^{l}}=\frac{1}{\Delta V_{0} \sqrt{g_{0}}} \int_{\Delta V_{0}} \overrightarrow{\tilde{g}}^{(l)} \cdot \vec{g}_{(k)} u^{k} H \sqrt{g_{0}} d \xi^{1} d \xi^{2} d \xi^{3}
\end{gathered}
$$

In order to solve the equations (3) and (4) we adopt a finite volume Shock-capturing scheme in which High-Order WENO reconstructions and the Exact Riemann solver differently from [910]. By using this numerical scheme, it is possible to calculate the point values of water depth and flow velocity at the centre of the cell faces of the computational grid.

\section{BOUNDARY CONDITION AT THE BOTTOM}

In this paper we adopt the governing equations proposed in [9-10] in which the Navier-Stokes equations are expressed in integral contravariant form in a time-dependent curvilinear coordinate system.

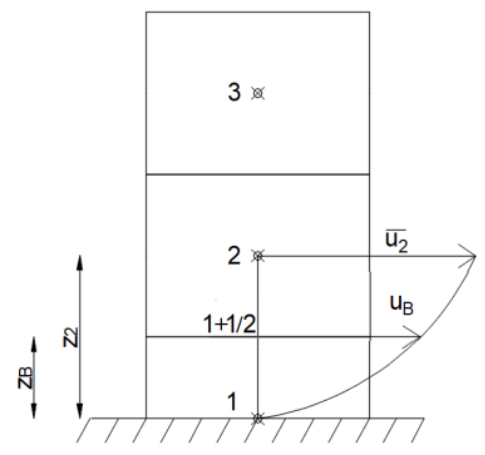

Figure 1 Computational grid cells at the bottom
The first grid cells near the bottom are shown in Figure 1.

For the sake of simplicity, let us consider flat bottom. We indicate with $z_{2}$ the distance from the bottom of the centre of the first calculation cell closest to the bottom (indicated with 2 in Figure 1) and with $z_{B}$ the distance from the bottom of the lower face of the first calculation cell closest to the bottom. The centre of the grid cell 1 belong to the bottom (as shown in Figure 1). $\overline{u_{2}}$ is the cartesian based velocity cell-averaged, placed at the centre of the cell 2, deducted from the contravariant components of the velocity, obtained by Equations (3) and (4).

The friction velocity $u^{*}$ is calculated by the following logarithmic law

$$
\frac{\overline{u_{2}}}{u^{*}}=\frac{1}{\kappa} \ln \left(\frac{E u^{*} z_{2}}{v}\right)
$$

where $E=0.9$ is a coefficient used for a smooth wall; $\kappa=0.41$ is the von Kármán constant and $v$ is the kinematic viscosity. The velocity boundary condition $u_{B}$ is calculated at the lower face of the calculation cell closest to the bottom point, that is at $(1+$ $1 / 2)$, by using the logarithmic law $\left(u=u^{*} / \kappa \ln \left(E u^{*} z_{B} / v\right)\right.$ ), through the friction velocity $u^{*}$.

As known, the turbulent boundary layer is divided in three regions: the viscous sub-layer is characterized only by the presence of the viscous stresses, $\left(y^{+} \leq 5\right.$ where $y^{+}=z u^{*} / v$ and $z$ is the distance from the wall in a Cartesian coordinate system); the buffer layer is characterized by the presence of the viscous stresses and turbulent stresses $\left(5<y^{+}<30\right)$; the turbulent core is characterized by the dominant presence of turbulent stresses $\left(30<y^{+}<100\right)$. The point at the centre of the calculation cell, in which the velocity is calculated, (in Figure 1 is the point (2), distant $z_{2}$ from the wall) needs to be placed on the turbulent core, because the logarithmic law is valid in the turbulent core.

The points $(1+1 / 2)$ and (2), during the wave period, are located out the oscillating turbulent wave boundary layer, or in the turbulent core, or in the buffer layer, or in the viscous sub-layer, because the in the $\sigma$-coordinate models, present in the literature, the points position $(1+1 / 2)$ and (2) oscillates consistent whit the free surface movement, as shown in Figure 2(a). By doing this, we determine wrong values of the velocity boundary condition and the turbulent stresses near the wall; indeed these values wrong evaluation of the phase-averaged crest elevations.

In order to correctly evaluate the phase-averaged crest elevations, we propose an analysis on the importance of the correct placement of the points $1(1+1 / 2)$ and (2)in the turbulent core.

The turbulent closure relation used in this paper, collocated on the lower face of the calculation cell closest to the bottom, is expressed as follows

$$
\tau_{m n}=2 v_{e f f} S_{m n}
$$

where $\tau_{m n}$ are the components of the turbulent stress tensor $(\bar{R})$; $v_{e f f}=v+v_{t}$ is the effective viscosity and $v_{t}$ is the turbulent eddy viscosity; $S_{m n}$ are the contravariant component of strain rate tensor.

The turbulent eddy viscosity is expressed by

$$
v_{t}=\kappa u^{*} z_{B}
$$

The Equation (9) is deducted by the hypothesis of the balance between the production and the dissipation of turbulent kinetic 
energy, that is holds true on the lower part of the turbulent core, near the buffer layer.

The turbulent eddy viscosity is evaluated by $v_{t}=$ $\left(C_{S} \Delta\right)^{2} \sqrt{2 S^{m n} S^{m n}}$ outside the turbulent boudary layer. Let $\Delta=$ $\sqrt[3]{\Delta \xi^{1} \Delta \xi^{2} \Delta \xi^{3}}$ be the length scale dependent on the grid size and $C_{s}$ be the Smagorinsky coefficient.

In this paper the point $(1+1 / 2)$ is located in the turbulent core near the buffer layer, where the balance between the production and dissipation of turbulent kinetic energy holds true; the point (2) is always located in the turbulent core. Both points oscillate coherently with the turbulent boundary layer, as shown in Figure 2(b).

In Figure 2(b) it is possible to notice that the first cell thickness increases in correspondence of the reduction of the velocity at the bottom and it reduces in correspondence of the increase of the velocity at the bottom.
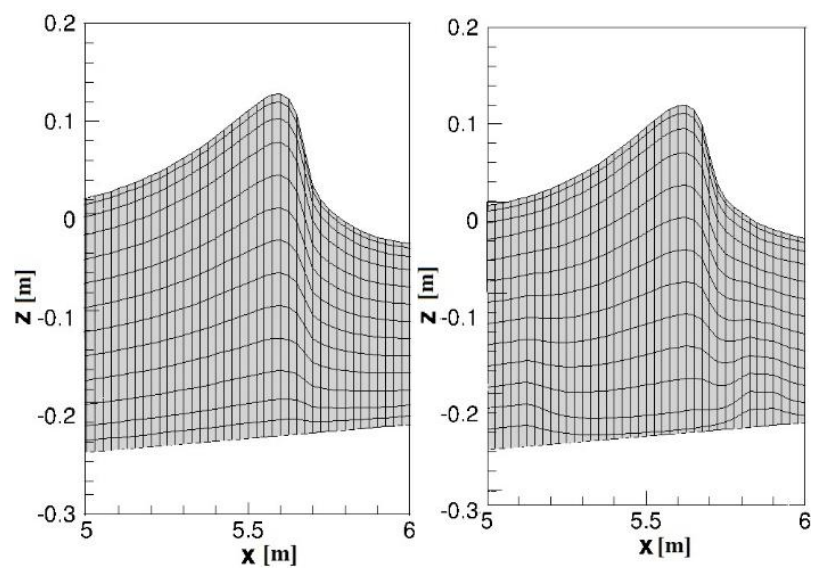

(a)

(b)

Figure 2 Instantaneous representation of: a) computational grid in which the points $1+1 / 2$ and 2 move whit the free surface; b) computational grid in which the points $1+1 / 2$ and 2 move with the oscillating wave boundary layer.

\section{RIP CURRENT TEST}

In this Section, in order to validate and verify the ability of the numerical model and to underline the importance of the correct location of the calculation cell closest to the bottom at $y^{+}=40$, we numerically reproduce wave propagation, wave breaking and hydrodynamic velocity fields. We reproduce a laboratory experiment carried out by Hamm [12].

The tank used by Hamm [12] measured $30 \mathrm{~m}$ by $30 \mathrm{~m}$; the sea bed consisted of a plane beach sloping at 1 in 30, with a rip channel excavated in the centre which produce a curved shaped coastline. The basin is symmetric with respect to the y-axis; for this reason it is sufficient to reproduce only one-half of the basin.

The turbulence stress tensor is estimated by the Smagorinsky subgrid model in which the Smagorinsky coefficient is set to 0.21 .

In Figure 3 a curvilinear computational grid and bottom variation, in which only one out of every five coordinate lines. We numerically reproduce a regular wave train with period $\mathrm{T}=1.25 \mathrm{~s}$ and height $\mathrm{H}=0.07 \mathrm{~m}$.
In Figure 4 a three-dimensional instantaneous wave field is shown. From this Figure it is possible to notice that the wave height increases in correspondence with the channel location due to occurrence of a pronounced rip current along the channel.

The time-average of the cross-shore velocity components calculated near the bottom along the rip channel is shown in Figure 5. From this Figure it is possible to notice the good agreement between numerical result and experimental measurements [12].

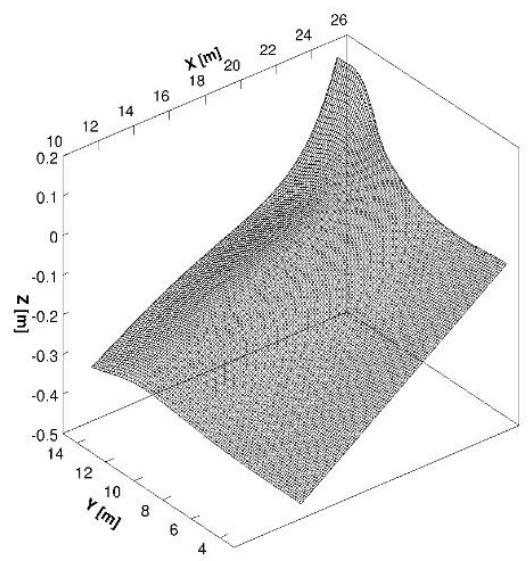

Figure 3 Three-dimensional view of the bottom and curvilinear computational grid (Only one out of every five coordinate lines is shown).

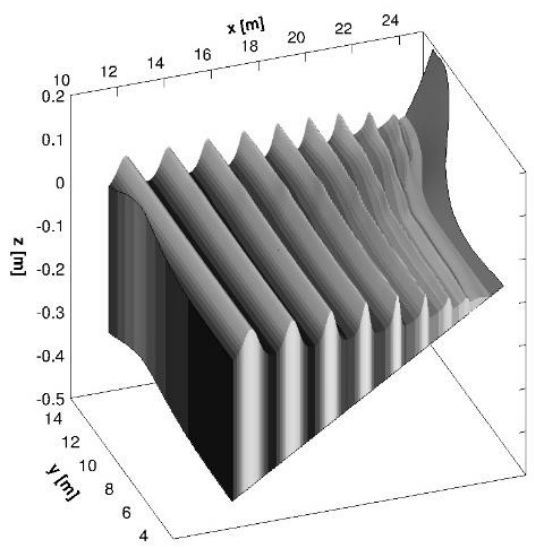

Figure 4 Three-dimensional view detail of an instantaneous wave field at the time when the breaking induced circulation is fully developed.

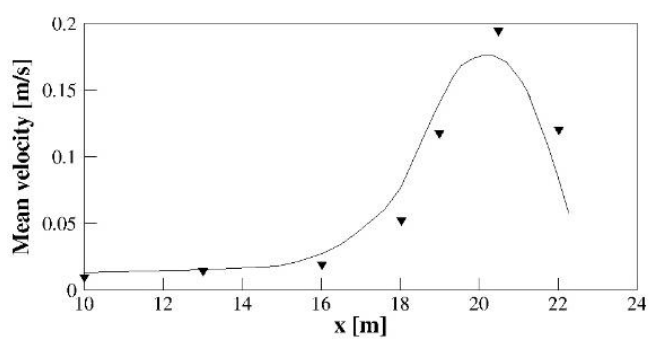

Figure 5 Mean current velocity along the rip channel.

Comparison between the experimental measurements [12] for unidirectional (triangles) random waves and the numerical results (solid line). 


\section{RESULTS}

In this Section we present the results of the numerical simulations of the wave breaking, obtained by the proposed model and we compare these results with the experimental data conducted by Ting and Kirby [13].

The experimental arrangement adopted by [13] consists in a sea bed with a sloping beach with slope 1:35. The still water depth is $h=0.4 m$. See Figure 6 for a schematic view.

The computational grid consists, for the numerical simulation, in: 13,728 grid cells in the horizontal direction with spacing $\Delta x_{1}=$ $0.025 \mathrm{~m} ; 13$ grid cells in the vertical direction. A cnoidal wave with period $T=2 s$ and wave height $H=0.125 m$, is imposed as input boundary condition.

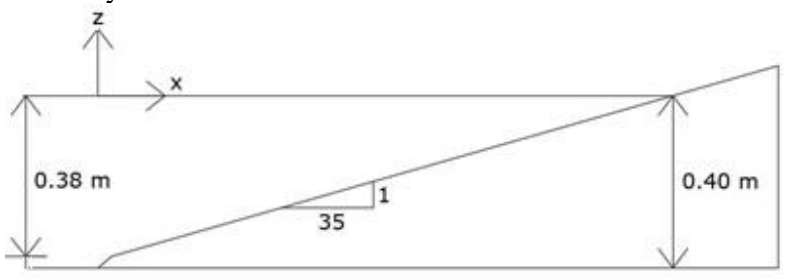

Figure 6 Schematic experimental arrangement by Ting and Kirby [13]

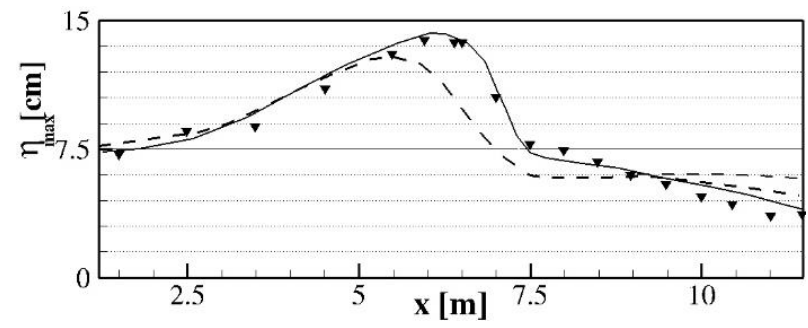

Figure 7 Ting and Kirby [13] breaking wave test case. Phaseaveraged crest elevations. Experimental data (circles) and numerical results obtained by $\boldsymbol{y}^{+}$average fixed value of 40 (dashed line) and $y^{+}$fixed value of 40 (solid line).

In Figure 7, the comparison between the results obtained with an oscillation of the lower face of the calculation cell closest to the bottom, with the free-surface movement (coherently with $\sigma$ coordinate models), and the results obtained with an oscillation of the aforementioned face, with the turbulent boundary layer, is shown.

The dashed line shows the cross-shore distribution of crest obtained by the numerical simulation in which the distance between the wall and the lower face of the calculation cell closest to the bottom oscillates with the free-surface movement and it is set to a dimensionless wall distance that has a time-averaged value equal to 40 .

The solid line shows the cross-shore distribution of crest obtained by the numerical simulation in which the distance between the wall and the lower face of the calculation cell closest to the bottom coherently oscillates with the turbulent boundary layer and it is always set to a dimensionless wall distance $y^{+}=40$, and it is always located inside the turbulent core, near the buffer layer, where the hypothesis of balance between production and dissipation of turbulent kinetic energy holds true.
In both the numerical simulations, outside the turbulent boundary layer, a Smagorinsky coefficient $C_{S}=0.21$ is adopted, in the turbulent closure relation.

The numerical results obtained with an oscillation of the lower face of the calculation cell closest to the bottom, with the freesurface movement (dashed line in Figure 7), show that the initial wave breaking point is located at $x=6.0 \mathrm{~m}$, much before the predicted location by the experimental results $(x=6.4 \mathrm{~m})$; from $x=4.8 \mathrm{~m}$ to $x=10.0 \mathrm{~m}$, the cross-shore distribution of crest is underestimated; after $x=10.0 \mathrm{~m}$, the cross-shore distribution of crest is overestimated.

The numerical results obtained with an oscillation of the lower face of the calculation cell closest to the bottom with the turbulent boundary layer (solid line in Figure 7) are in good agreement with the experimental measurement.

From the comparison between the results obtained with an oscillation of the lower face of the calculation cell closest to the bottom, with the free-surface movement, and the results obtained with an oscillation of the aforementioned face with the turbulent boundary layer, it is possible to notice the limitation of the $\sigma$ coordinate models that are present in the literature.

In the $\sigma$-coordinate models, the lower face of the calculation cell closest to the bottom follows the free-surface movement, in a contradictory oscillation with respect to the one of the turbulent boundary layer. Consequently, in the $\sigma$-coordinate models, the lower face of the calculation cell closest to the bottom, during the wave period, may be located alternatively outside the turbulent boundary layer, inside the turbulent core, inside the buffer layer, or (when the velocity is low) inside the viscous sub-layer. By using these models, the velocity boundary condition, the friction velocity and the turbulent bottom stresses are wrong evaluated, consequently there is an erroneous simulation of the cross-shore distribution of crest elevations.

The cell-averaged velocity value is calculated at the centre of the calculation cell closest to the bottom; by means of this cellaveraged velocity value, through the logarithmic law defined in Section 3 , the friction velocity $u^{*}$ and the velocity value at the lower face of the grid cell $u_{B}$, are computed.

The adopted logarithmic law holds true in the turbulent core. The turbulent eddy viscosity in the turbulent boudary layer is calculated by the equation presented previously, that is valid in the turbulent core near the buffer layer. The necessity of letting the centre of the aforementioned grid cell oscillate coherently with the turbulent boundary layer and the necessity of letting the lower face of the aforementioned grid cell be located near the buffer layer, have been demonstrated by the accordance between the numerical results and the experimental measurements, in terms of cross-shore distribution of crest and of location of the initial wave breaking point. 


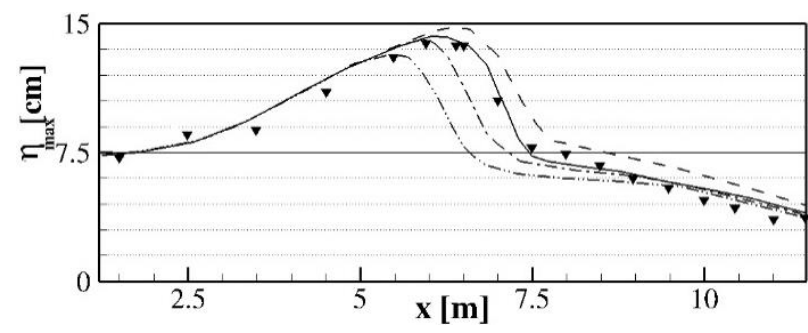

Figure 8 Ting and Kirby [12] breaking wave test case. Phaseaveraged crest elevation. Experimental data (circles) and numerical results with $y^{+}=80$ (dash dot dot line), $y^{+}=60$ (dash dot line), $y^{+}=40$ (solid line) and $y^{+}=30$ (dashed line).

In Figure 8, the comparison among the results obtained with four numerical simulations, in which the lower face of the calculation cell closest to the bottom oscillates with the turbulent boundary layer, is shown. In Figure 10, the cross-shore distribution of crest, obtained by the aforementioned four different simulations in which the distance between the wall and the lower face of the calculation cell closest to the bottom is set to a dimensionless wall distance $y^{+}=80$ (dash dot dot line), $y^{+}=60$ (dash dot line), $y^{+}=40$ (solid line) and $y^{+}=30$ (dotted line), is shown. In all the numerical simulations, the boundary condition proposed in this work is adopted. In the closure relation, outside the turbulent boundary layer, the Smagorinsky coefficient is set to $C_{S}=0.21$.

The numerical results obtained with the numerical simulation carried out with $y^{+}=80$ (dash dot dot line), show that the initial wave breaking point is located at $x=5.75 \mathrm{~m}$, much before the predicted location by the experimental results $(x=6.4 \mathrm{~m})$; from $x=5.75 \mathrm{~m}$ to $x=9.5 \mathrm{~m}$, the cross-shore distribution of crest is underestimated and their slope is lower than the one obtained by the experimental measurements. The numerical results obtained with the numerical simulation carried out with $y^{+}=60$ (dash dot line), show that the initial wave breaking point is located at $x=$ $6.25 \mathrm{~m}$, before the predicted location by the experimental results $(x=6.4 \mathrm{~m})$; from $x=6.25 \mathrm{~m}$ to $x=9.0 \mathrm{~m}$, the cross-shore distribution of crest is underestimated and their slope is comparable to the one obtained by the experimental measurements; after $x=9.0 \mathrm{~m}$, the cross-shore distribution of crest is slightly overestimated. The numerical results obtained with the numerical simulation carried out with $y^{+}=40$ (solid line), have been already described and show the best agreement with the experimental results. The numerical results obtained with the numerical simulation carried out with $y^{+}=30$ (dashed line), show that the initial wave breaking point is located at $x=6.5 \mathrm{~m}$, slightly after the predicted location by the experimental results $(x=6.4 \mathrm{~m})$; after $x=6.5 \mathrm{~m}$, the cross-shore distribution of crest is overestimated and their slope is higher than the one obtained by the experimental measurements. The importance to correctly locate the lower face of the calculation cell closest to the bottom is demonstrated by the comparison among the different simulations carried out. If the distance $z_{B}$ between the wall and the lower face of the calculation cell closest to the bottom increases, the friction velocity $u^{*}$ decreases; the velocity boundary condition $u_{B}$ decreases; the bottom turbulent stresses decrease, causing a decrease in the value of the cross-shore distribution of crest and an anticipation of the initial wave breaking point.

The necessity to letting the lower face of the calculation cell closest to the bottom (where the turbulent eddy viscosity $v_{t}$ is evaluated) oscillate at the lower face of the calculation cell closest to the bottom (where the turbulent eddy viscosity $v_{t}$ is evaluated), has been demonstrated by the accordance of the numerical results (solid line in Figure 8) with the experimental measurements in terms of cross-shore distribution of crest elevations and of location of the initial wave breaking point.

\section{CONCLUSIONS}

In this paper we propose a new numerical model for the simulation of the wave breaking. The three-dimensional equations are expressed in contravariant form and are solved over a curvilinear boundary conforming grid, which is capable to represent the complex geometries, typically present in coastal regions. A transformation over the time of the vertical coordinate, as a function of the movement of the oscillating turbulent boundary layer, other than the free-surface elevation. It has been demonstrated that the proposed numerical procedure allows us to correctly simulate the cross-shore distribution of crest and the location of the initial wave breaking point. It has been demonstrated that the centre of the first calculation cell must oscillate coherently with the turbulent boundary layer. It has been demonstrated that the lower face of the calculation grid cell, where the turbulent eddy viscosity $v_{t}$ is evaluated, must always oscillate inside the turbulent core and near the buffer layer, where the hypothesis of balance between production and dissipation of turbulent kinetic energy holds true.

\section{REFERENCES}

[1] Cannata, G., Barsi, L., Petrelli, C. and Gallerano, F. 2018. Numerical investigation of wave fields and currents in a coastal engineering case study. WSEAS Transactions on Fluid Mechanics. 13, 87-94.

[2] Shi, F., Kirby, J.T., Harris J.C., Geiman, J.D. and Grilli, S.T. 2012. A high-order adaptive time-stepping TVD solver for Boussinesq modelling of breaking waves and coastal inundation. Ocean Model. 43-44, 36-51.

[3] Cioffi, F., F. and Gallerano F. 2006. From rooted to floating vegetal species in lagoons as a consequence of the increase of external nutrient load: An analysis by model of the species selection mechanism. Applied Mathematical Modelling. 30(1), 10-37.

[4] Gallerano, F., Cannata, G., and Scarpone, S. 2017. Bottom changes in coastal areas with complex shorelines. Engineering Applications of Computational Fluid Mechanics. 11(1), 396-416.

[5] Caleffi, V., Valiani, A. and Li, G. 2016. A comparison between bottom-discontinuity numerical treatments in the DG framework. Applied Mathematical Modelling. 40, 75167531.

[6] Ma, G., Shi, F. and Kirby, J.T. 2012. Shock-capturing nonhydrostatic model for fully dispersive surface wave processes. Ocean Modelling. 43-44, 22-35.

[7] Bradford, S.F. 2011. Non-hydrostatic model for surf-zone simulation. Journal of Waterway, Port, Coastal, and Ocean Engineering. 137, 163-174.

[8] Cannata, G., Gallerano, F., Palleschi, F., Petrelli, C. and Barsi, L. 2019. Three-dimensional numerical simulation of the velocity fields induced by submerged breakwaters. International Journal of Mechanics. 13, 1-14.

[9] Palleschi, F., Iele, B. and Gallerano, F. 2019. Integral contravariant form of the Navier-Stokes equations. WSEAS Transactions on Fluid Mechanics. 14, 101-113. 
[10] Cannata, G., Petrelli, C., Barsi, L. and Gallerano, F. 2019. Numerical integration of the contravariant integral form of the Navier-Stokes equations in time-dependent curvilinear coordinate systems for three-dimensional free surface flows. Continuum Mechanics and Thermodynamics. 31, 491-519.

[11] Luo, H., and Bewley, T. R. 2004. On the contravariant form of the Navier-Stokes equations in time dependent curvilinear coordinate systems. Journal of Computational Physics. 199, 355-375.

[12] Hamm, L. 1992. Directional nearshore wave propagation over a rip channel: an experiment. Proceedings of the 23rd International Conference of Coastal Engineering, 226-239.

[13] Ting, F.C.K. and Kirby, J.T. 1994. Observation of undertow and turbulence in a laboratory surf zone. Costal Engineering. 24, 51-80 